\title{
Performance Characterization of the Low-Power Halo Electric Propulsion System
}

\author{
C. Ryan* \\ University of Southampton, Southampton, England SO17 1BJ, United Kingdom \\ and \\ T. Wantock,, \pm T. Harle,, \pm and A. Knollı \\ University of Surrey, Guildford, Surrey GU2 7XH, United Kingdom
}

\begin{abstract}
DOI: $10.2514 / 1 . B 36091$
Performance measurements have been obtained of a novel propulsion concept called the Halo thruster under development within the University of Surrey. The Halo thruster, a type of cusped-field thruster with close similarity to the cylindrical Hall thruster, is motivated by the need for low-power and low-cost electric propulsion for the small satellite sector. Two versions of the device are investigated in this study: a design using permanent magnets at high magnetic-field strength and a design using electromagnets with moderate field strength. While operating at $200 \mathrm{~W}$ discharge power, which is of particular interest to power-limited small satellite platforms, the permanent-magnet design achieved a maximum thrust efficiency of $8 \%$ at a specific impulse of approximately $900 \mathrm{~s}$ using a krypton propellant. By comparison, the electromagnet design achieved a maximum thrust efficiency of $28 \%$ at a specific impulse of approximately $1500 \mathrm{~s}$ at $200 \mathrm{~W}$ using a xenon propellant. For higher levels of power (tested up to $800 \mathrm{~W}$ ), the performance of the electromagnetic design saturated at approximately $25 \%$ thrust efficiency using krypton and $30 \%$ using xenon. The thrust efficiency of the permanent-magnet design appeared to increase monotonically up to $600 \mathrm{~W}$ reaching a maximum value of $14 \%$.
\end{abstract}

\section{Introduction}

$\mathbf{T}$ HE Halo thruster, a concept under development at the University of Surrey, was primarily motivated to offer a low-cost electric propulsion (EP) system that could operate at power levels available on small satellites. More typical EP devices [Hall-effect thrusters (HETs), gridded ion thrusters] do not operate well at power requirements of several hundred watts. For example, HETs encounter problems when scaled down to smaller sizes due to practical issues related to the higher levels of magnetic field required, and fundamental issues related to the plasma volume vs the surface loss area. This has resulted in an interest in EP devices derived from the HET, which can scale to lower powers more efficiently. One such alternative is an HET-like thruster that uses cusp magnetic-field structures. Cusp magnetic structures are used in cylindrical Hall thruster (CHT) [1, 2] diverging cusped-field thrusters [3, 4$]$, and the high-efficiency multistage plasma (HEMP) thruster [ $[-8-8]$. These devices employ magnetic cusps to promote mirror confinement of electrons, which reduces wall losses and promotes increased ionization efficiency at low powers. The ions created by the electron bombardment are accelerated by the axial electric field resulting from the electron magnetic confinement, exiting the thruster with a large axial-velocity component.

The Halo thruster was originally conceived as a permanent-magnet (PM) design. It features a cusped magnetic field similar to that of the CHT, but with the novel addition of a toroidal cusp layer close to the thruster anode, produced by two concentric PMs. This resulted in a large number of magnetic cusps within the thruster channel, which is thought to promote greater electron confinement. Utilizing only two PMs resulted in four axisymmetric cusps being created (Fig. 1).

Received 11 November 2015; revision received 4 April 2016; accepted for publication 26 June 2016; published online XX epubMonth XXXX. Copyright $\odot 2016$ by the American Institute of Aeronautics and Astronautics, Inc. All rights reserved. Copies of this paper may be made for personal and internal use, on condition that the copier pay the per-copy fee to the Copyright Clearance Center (CCC). All requests for copying and permission to reprint should be submitted to CCC at www.copyright.com; employ the ISSN 07484658 (print) or 1533-3876 (online) to initiate your request.

*Astronautics Research Group, Faculty of Engineering and the Environment.

${ }^{\dagger}$ Surrey Space Centre.

†Surrey Space Centre; a.knoll@surrey.ac.uk (Corresponding Author).
Secondly, the cusp structure above the anode allowed for the electrons to reach the anode if they possessed enough kinetic energy break through the magnetic mirror. This differs from an HET, in which the electrons reach the anode through anomalous Bohm diffusion. It was hoped that this would reduce the voltage requirements for the anode power supply, which can approach many hundreds of volts for CHTs.

As a result of the cusp configuration, an annulus of magnetic-field cancellation within the discharge channel was created. This "halo" of magnetic cancellation, enclosed on all sides by magnetic cusps, gives the thruster its name.

An electromagnetic (EM) Halo thruster has also been developed with a similar magnetic structure, but with greater similarity to a CHT (Fig. 3). The motivation was again to produce an EP thruster that could operate well at low powers. A similar Halo ring of magnetic cancellation was included within the design, resulting in a concentrated cusp field structure. The cusps were also very close to the anode, with the hope that this would promote the electron path toward the anode, hence reducing the anode voltage.

This paper presents experimental measurements of the PM and electromagnet variants of the Halo thruster, detailing its performance in terms of current and voltage, and thrust, $I_{\mathrm{sp}}$ measurements using a pendulum-type thrust balance. Firstly, the Halo thruster is described, both in terms of its magnetic configuration and general design. Details of the experimental design and method are provided, including vacuum-chamber setup and thrust-balance design.

\section{PM Halo Thruster Design}

The PM Halo thruster design is illustrated in Fig. 1. The PM Halo 8 thruster consists of two annular neodymium-iron-boron PMs of identical axial polarity aligned concentrically. This configuration results in four axisymmetric cusp regions within the thruster, and an annular region of magnetic cancellation near the anode. This annular region of magnetic cancellation, and the associated magnetic cusps around it, it was hoped, would result in it being easier for the electrons to reach the anode, and therefore, result in a lower anode voltage.

The thruster channel is constructed from boron nitride with a cylindrical and annular part of axial lengths 23 and $20 \mathrm{~mm}$, respectively. The annular portion of the thruster has an inner diameter of $38.5 \mathrm{~mm}$ and an outer diameter of $46.5 \mathrm{~mm}$. Utilizing approximately half the annular volume is a $20-\mathrm{mm}$-long copper anode formed from a 


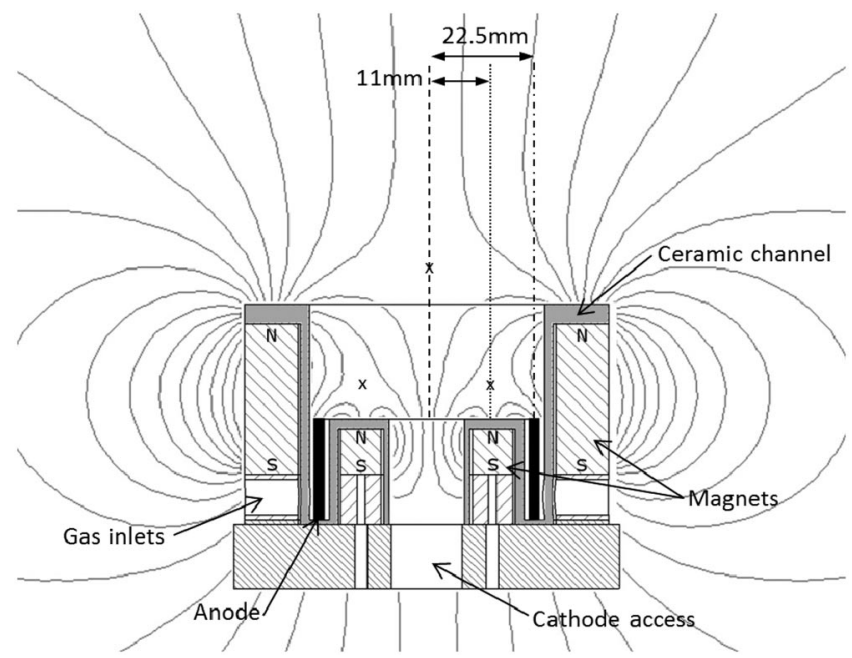

Fig. 1 Design and magnetic profile of the PM Halo thruster.

41-mm-inner-diam cylinder $1.6 \mathrm{~mm}$ thick. The propellant gas is fed from an inlet within the annular section of the thruster on the outer channel wall.

Along the central axis of the thruster is a 15-mm-diam cavity, in which a cathode neutralizer is situated, in a design similar to that found in early HEMP thrusters [8]. A commercial hollow cathode (HeatWave model HWPES-250) was used for low-power operation, and an RF plasma-bridge neutralizer [9] was used for high-power conditions. The RF neutralizer design was derived from the nonambipolar electron source developed by Longmier et al. [10]. It consists of a Pyrex source tube with a 5-mm-diam exit orifice, within which a stainless-steel ion collection cylinder is contained. Eight equidistant axial slots of $60 \mathrm{~mm}$ length are cut into the ion collection cylinder to mitigate against coupling with a double-loop antenna, which sits concentrically with the source tube. Gas is fed into the device along the central axis. In contrast to the nonambipolar electron source, no magnetic field is applied and no grounded ring at the orifice is employed; instead, ions are lost through thermal diffusion to the grounded ion collection cylinder, allowing electrons to escape through the orifice to an external anode. The device has been measured to deliver up to $4 \mathrm{~A}$ to the thruster anode with fixed conditions of $200 \mathrm{~W}$ RF power and 4 standard cubic centimeters per minute (sccm) krypton flow.

Figure $\underline{2}$ displays the simulated total magnetic-field profile of the PM Halo thruster displayed in Fig. 1, along the central axis, an axial path intersecting the halo null region at $11 \mathrm{~mm}$ radial distance from

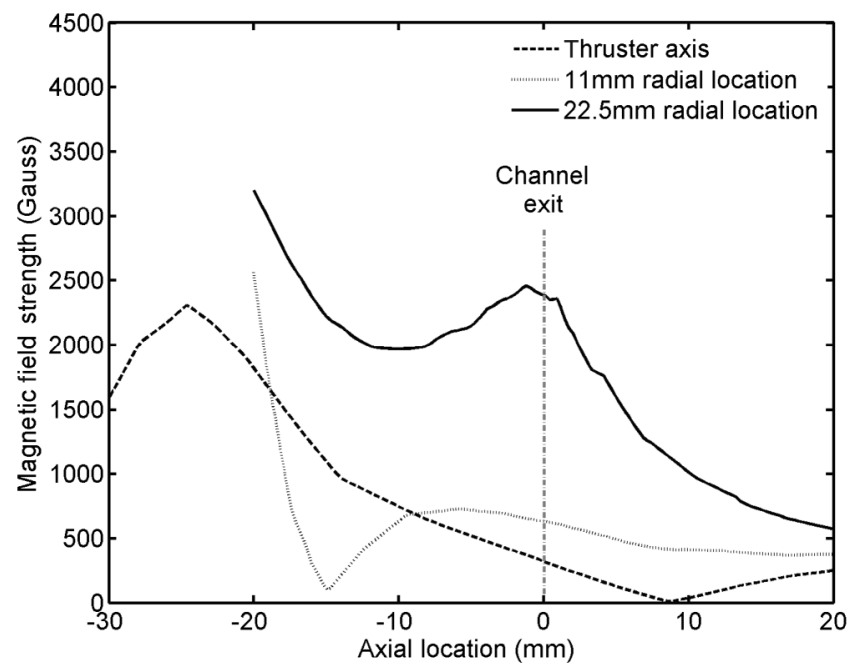

Fig. 2 Simulated total magnetic-field-strength profile along the central axis, along an axial path through the halo null region, and along an axial path from the anode. the central axis, and along an axial path from the top surface of the anode at $22.5 \mathrm{~m}$ radial distance from the central axis. The halo magnetic-cancellation region is visible at approximately $1 \mathrm{~cm}$ in front of the anode. Owing to the use of strong PMs, the magnetic-field strength is large, especially along the channel walls where it peaks at above $0.3 \mathrm{~T}(3000 \mathrm{G})$.

\section{Electromagnet Halo Thruster Design}

Figure 3 illustrates the magnetic profile and design of the EM Halo thruster. It is similar to the PM Halo thruster with an annular magneticcancellation region near the anode resulting in a large number of cusps, promoting electron confinement while also making it easier for the electrons to reach the anode. It also has the same $50 \mathrm{~mm}$ channel diameter as the PM Halo thruster. The EM Halo thruster employs four annular electromagnets of two different sizes aligned along the outer diameter of the discharge channel, instead of two PMs. The polarity of the two smaller electromagnets is configured to be in opposition to the two larger electromagnets. A magnetic circuit produces a magneticfield topology with a strong radial field component between two regions of magnetic cancellation. The magnetic circuit consists of a ferromagnetic steel cylinder enclosing the electromagnets and thruster channel; several 2-mm-thick ferromagnetic plates between, above, and below the electromagnets; and a 7.25-mm-diam central-pole piece. The use of a thick central pole results in annular and cylindrical sections of the thruster, similar to that of early designs of CHTs [1]. The axial length of the annular section of the thruster is $14 \mathrm{~mm}$, and the cylindrical section is $35 \mathrm{~mm}$. The annular section has an inner diameter of $20 \mathrm{~mm}$.

A stainless-steel anode is situated in the annular part of the discharge channel. Propellant gas is fed through $102-\mathrm{mm}$-diam holes in the anode's top face. To initiate the discharge, $300 \mathrm{~V}$ is applied to the anode relative to the grounded cathode mounted downstream of the channel exit. The cathode is mounted perpendicular to the thruster axis, with its orifice located $20 \mathrm{~mm}$ downstream of the thruster exit and $30 \mathrm{~mm}$ from the thruster axis. The assembled thruster has a mass of $2.2 \mathrm{~kg}$ and a height of $74 \mathrm{~mm}$.

Figure 4 displays the simulated total magnetic-field profile of the EM Halo thruster along the central axis, an axial path from the inner radius of the anode at $10 \mathrm{~mm}$, and an axial path from the outer radius of the anode at $25 \mathrm{~mm}$. The halo-shaped magnetic-cancellation region is visible at approximately $5 \mathrm{~mm}$ in front of the anode. The magnetic-field strength peaks at around $900 \mathrm{G}$ close to the magneticpole piece on the central axis.

\section{EM Halo Thruster Experimental Setup}

Thrust-balance performance characterization was carried out in the Electric Propulsion Laboratory of the Surrey Space Centre, University of Surrey. The measurements were completed in the Daedalus vacuum-chamber facility, a 1.5 -m-diam by 3-m-long chamber equipped with turbomolecular and cryogenic pumps.

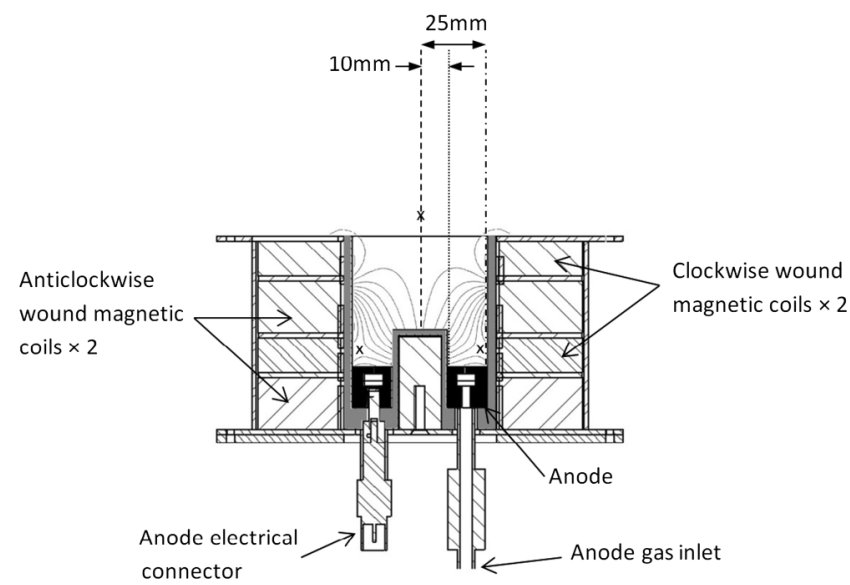

Fig. 3 Design of the EM Halo thruster, including magnetic profile. 


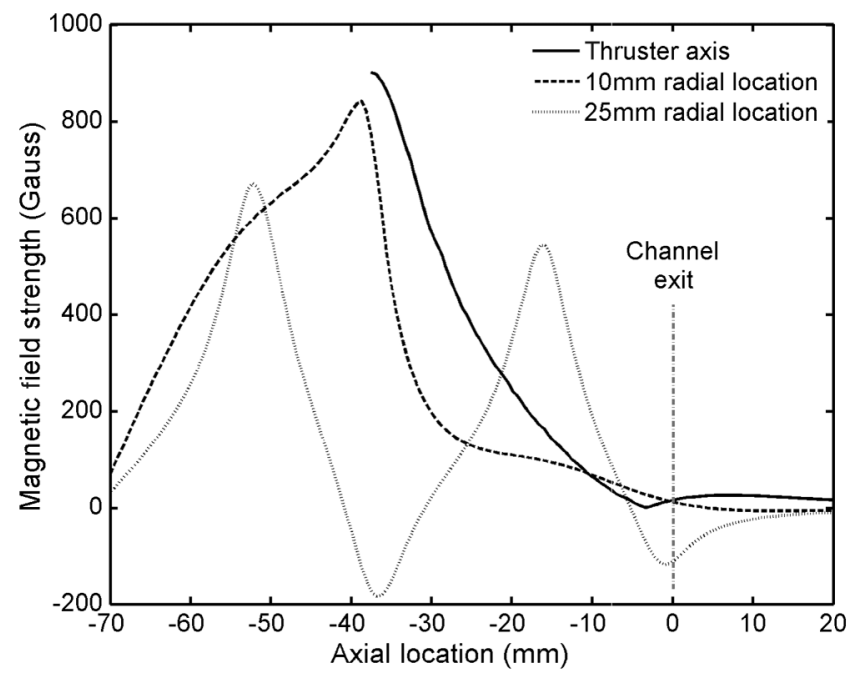

Fig. 4 Simulated profiles of magnetic-field strength along three axial paths for $2 \mathrm{~A}$ current to the electromagnets.

The experiment made use of a pendulum thrust balance, a technique employed extensively in other studies $[16,17]$. The balance used consists of a swinging platform to which a thruster is mounted. This platform is attached to the stationary frame above it by aluminum flexures, allowing it to freely swing horizontally. The displacement of the balance is measured using a Micro-Epsilon IDL1700-2 optical triangulation laser system, with a resolution of $0.1 \mu \mathrm{m}$.

Krypton gas was predominantly used, with some measurements using xenon. A hollow cathode developed in-house at the Surrey Space Centre was used [18], operating with a keeper current of $1.5 \mathrm{~A}$. To avoid excessively heating the balance, the hollow cathode was not attached to the thrust balance with the thruster. The volumetric flow rate to the cathode was kept fixed at $10 \mathrm{sccm}$, whereas to the thruster anode it was varied between 5,10 , and $15 \mathrm{sccm}$. The gas-flow rate to the cathode was kept relatively high as the laboratory cathode available at the time operated best at these higher flow rates. Given that the cathode operation is unrealistic for flight operation, it was not taken into account in calculations of specific impulse and thrust efficiency.

Before each measurement, gas flow to the anode was delivered via an MKS Type 247 mass flow controller, and $300 \mathrm{~V}$ relative to the cathode was applied to the anode, initiating a glow discharge. The electromagnets were then turned on and the anode power set by varying the discharge current, before displacement data from the laser were acquired for $20 \mathrm{~s}$. After measuring the displacement of the balance with the thruster turned on, the anode power supply was then turned off, and displacement data were acquired for another $20 \mathrm{~s}$. The change in average displacement as a result of the thruster's operation was then used to obtain thrust, using the balance's measured calibration factor.

\section{Current-Voltage Characteristics}

The variation of the anode voltage and current, given by the displayed values on the anode power supply, was noted across the powers, flow rates, and electromagnet currents tested.

Figure 5 illustrates the variation of current-voltage characteristics of the Halo thruster at different magnetic-field-strength conditions, using krypton at a fixed anode flow rate of $10 \mathrm{sccm}$. The current to the four electromagnets was varied from 0.5 to $2 \mathrm{~A}$. The Sorensen highvoltage power supply used (DCS 300-4E) could output a maximum current and voltage of $4.2 \mathrm{~A}$ and $304 \mathrm{~V}$, respectively.

The data of Fig. 5 indicate a change in the behavior of the thruster at higher power. Above approximately $100 \mathrm{~V}$, the rate of change of the discharge current with increasing voltage decreases, suggesting a change in the mode of operation of the thruster. This behavior is a characteristic of the current saturation observed in other Hall-effect devices. The gradient of the $I-V$ characteristic curve decreases with

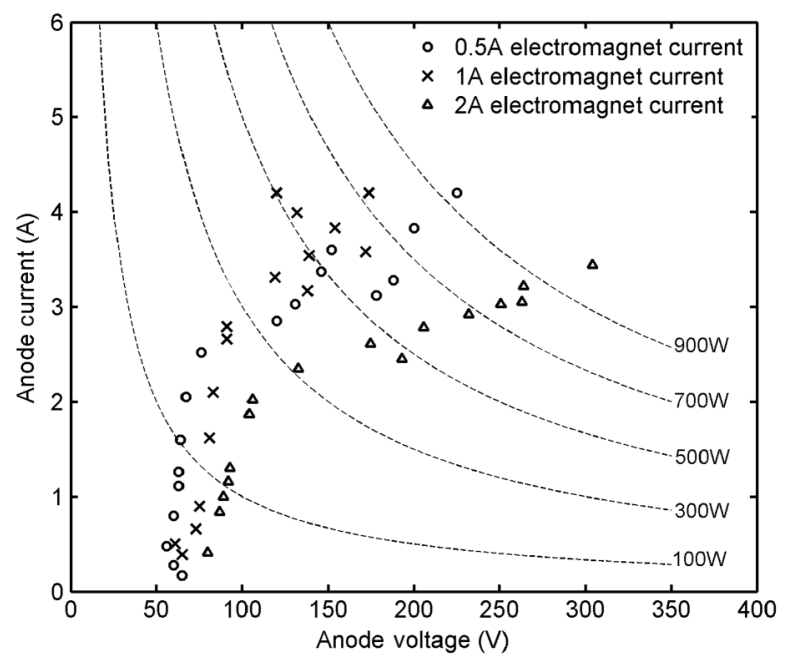

Fig. 5 Current vs voltage trends for the EM Halo thruster using a krypton propellant at an anode flow rate of $10 \mathrm{sccm}$.

increasing magnetic-field strength, and the inflection point of the curve occurs at higher voltages with increasing magnetic-field strength.

Figure 6 displays the current-voltage characteristics for the EM and PM Halo thrusters at various volumetric flow rates. Current saturation can again be inferred by the presence of inflection points along each curve.

\section{Performance Characteristics}

\section{A. PM Halo Thruster}

Figure 7 displays thrust-measurement data as a function of anode power for the PM Halo thruster, using the hollow cathode at the lowest anode flow rate $(2 \mathrm{sccm})$ and the RF electron source at other flow rates. The error on the thrust, specific impulse, and thrust efficiency, from the standard deviation of at least six repetitions, was found to be relatively consistent. Values of $0.4 \mathrm{mN}, 50 \mathrm{~s}$, and $1 \%$, respectively, were found. The PM Halo thruster study using the hollow cathode focused on performance at low flow rates and low anode powers. The sensitivity of the thruster to various flow-rate combinations was first mapped out at very low anode powers (10$20 \mathrm{~W}$ ), before a power sweep, using 2 sccm krypton to both anode and 1 hollow cathode, was taken up to $100 \mathrm{~W}$. The results of this initial campaign demonstrated some potential for the concept - at $100 \mathrm{~W}$ anode power and $2 \mathrm{sccm}$ of krypton, the thruster delivered thrust of

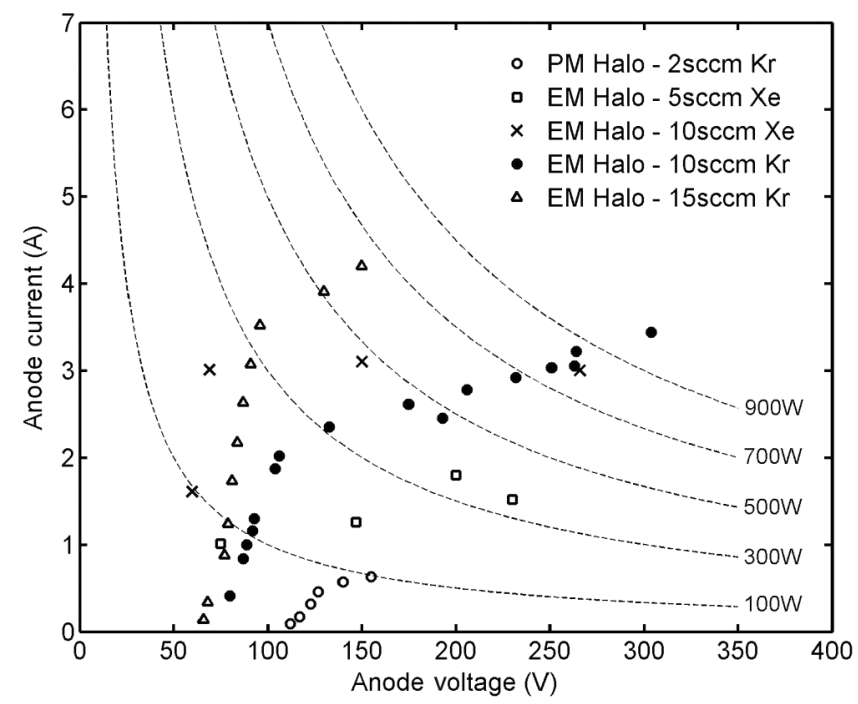

Fig. 6 Comparison between current-voltage trends for the PM and EM Halo thrusters at different volumetric flow rates of krypton and xenon propellants; the electromagnet current was kept fixed at $2 \mathrm{~A}$. 
$3 \mathrm{mN}, I_{\mathrm{sp}}$ of $1100 \mathrm{~s}$, and anode efficiency of $10 \%$. These results encouraged further investigation of the proof-of-concept prototype at higher powers and flow rates; this provided motivation for the development of an RF electron source. For the PM Halo thruster using the RF electron source, measurements of thrust were made at anode flow rates of 7, 10, and $15 \mathrm{sccm}$ (Fig. 7), with the RF electron source operating at a $4 \mathrm{sccm} \mathrm{Kr}$ flow rate throughout. The resulting data indicated that the PM prototype also operates favorably at higher

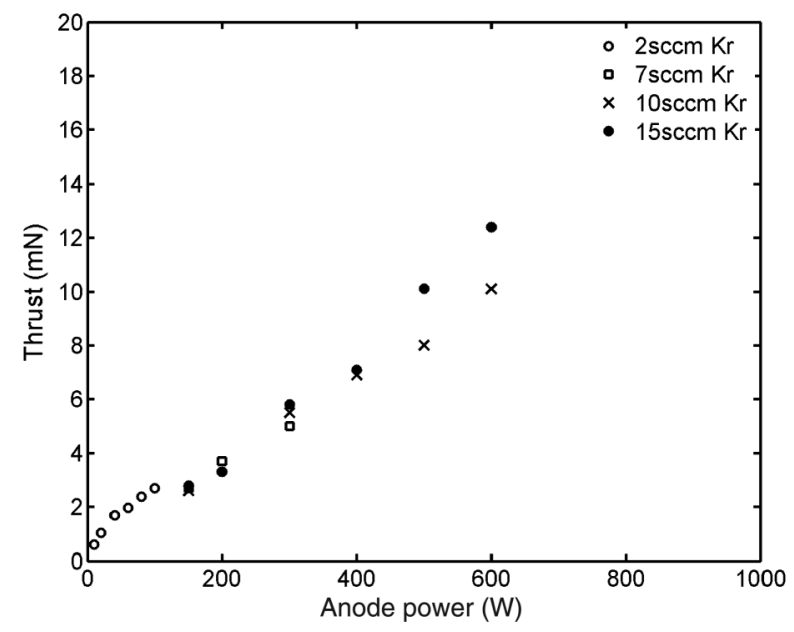

a)

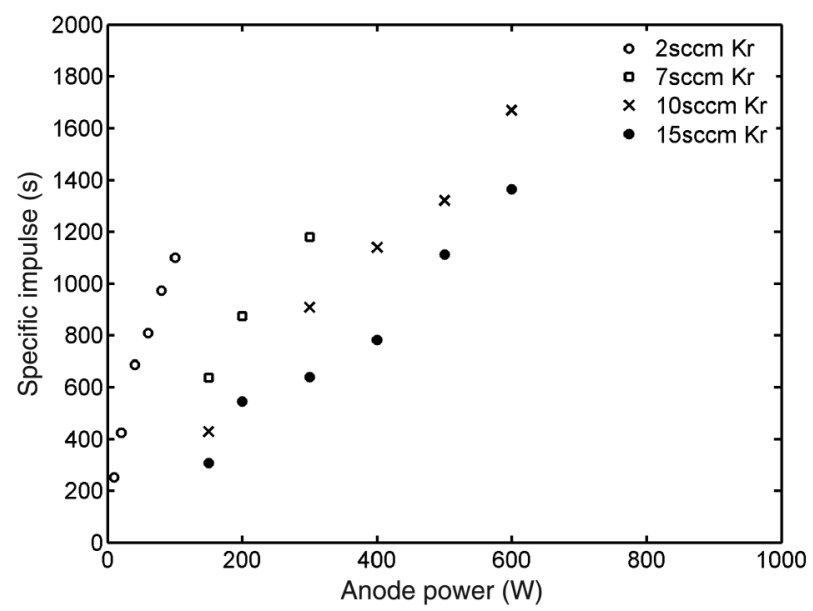

b)

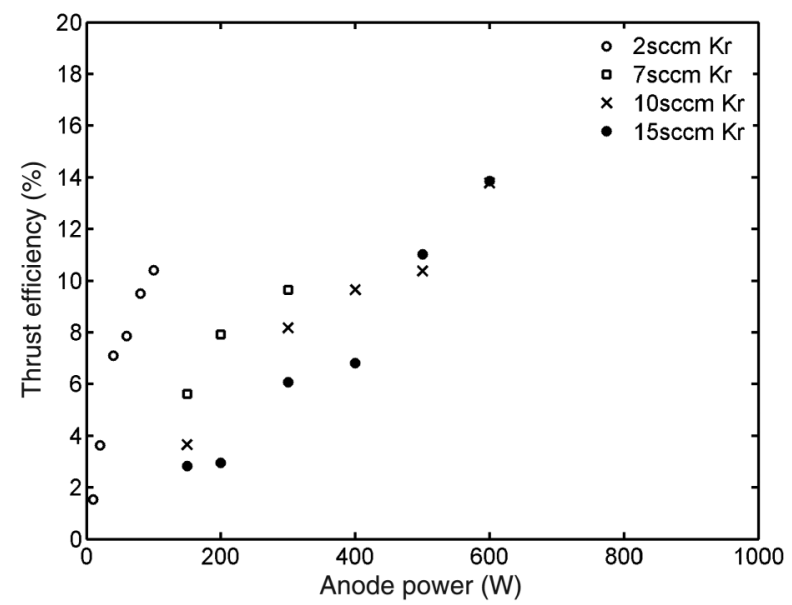

c)

Fig. 7 Preliminary results of thrust-balance performance characterization for the PM Halo thruster variants, for several anode flow rates of krypton; 2 sccm flow rate used a hollow cathode; the remainder used the RF neutralizer. flow rates and powers, with measured performance of thrust $\sim 10 \mathrm{mN}$ $I_{\mathrm{sp}} \sim 1600 \mathrm{~s}$, and anode efficiency $\sim 14 \%$ at $600 \mathrm{~W}$ and $10 \mathrm{sccm}$ krypton anode flow rate. There is no plateau in performance apparent in the RF Halo data, suggesting further improvement at higher powers. The discharge became unstable at powers beyond $300 \mathrm{~W}$ for the $7 \mathrm{sccm}$ case. For each data point displayed in Fig. 7, the nominal systematic uncertainty due to thrust-balance resolution is of order $0.1 \mathrm{mN}$. Further experiments are required to characterize the proofof-concept prototype beyond these preliminary measurements.

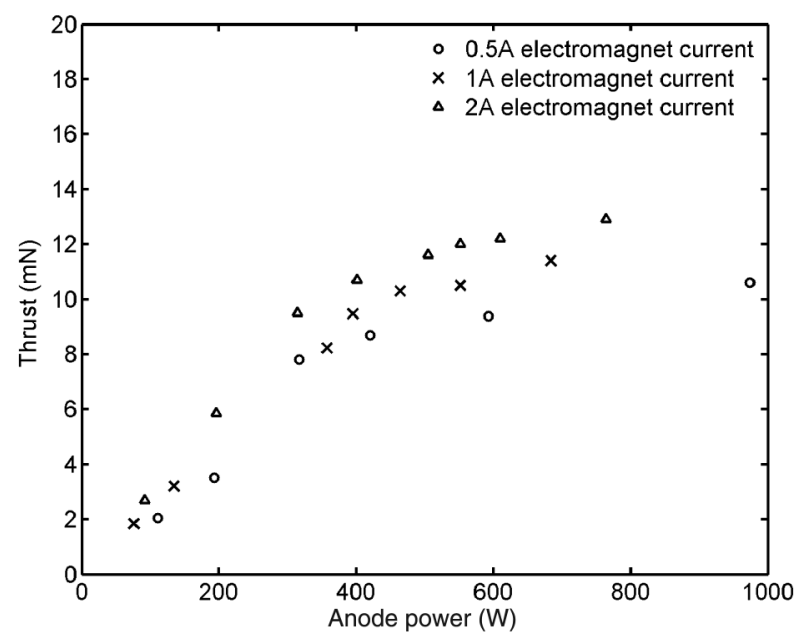

a)

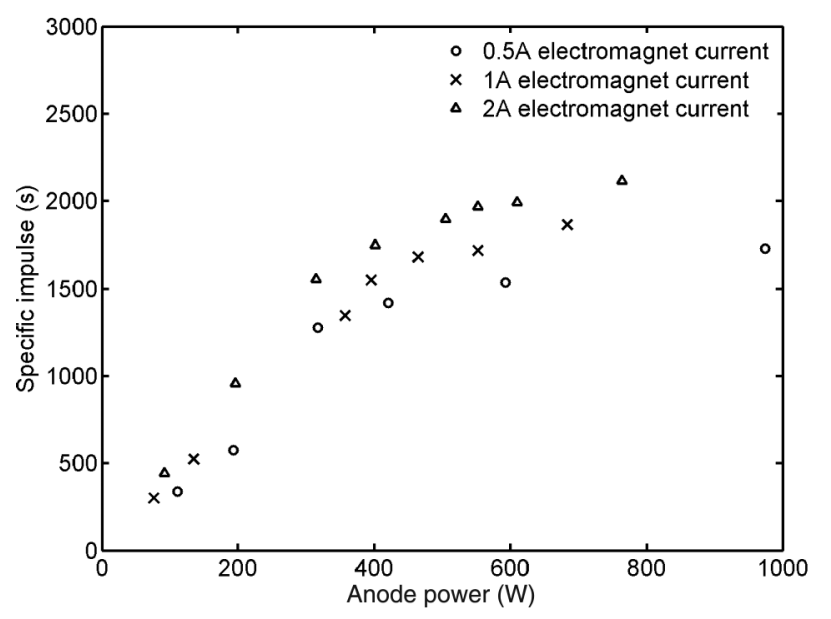

b)

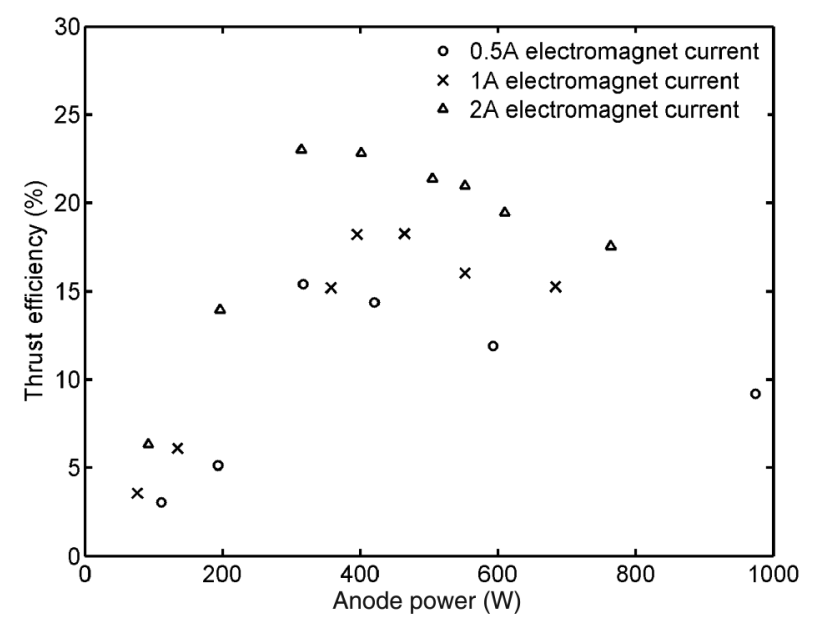

c)

Fig. 8 Performance values of the EM Halo thruster with power using krypton, at an anode flow rate of $10 \mathrm{sccm}$. 


\section{B. Electromagnetic Halo Thruster}

The performance measurements of the EM thruster were completed at flow rates of 5,10 , and $15 \mathrm{sccm}$ using krypton, and 5 and $10 \mathrm{sccm}$ using xenon. The current to the four electromagnets was varied between $0.5,1$, and $2 \mathrm{~A}$.

Figure 8 illustrates the variation of measured thrust, specific impulse, and anode efficiency with anode power at an anode flow rate of krypton $10 \mathrm{sccm}$. Each data point is an average of six thrust measurements, with the power calculated from the measured current and voltage applied to the anode. The thrust and specific impulse

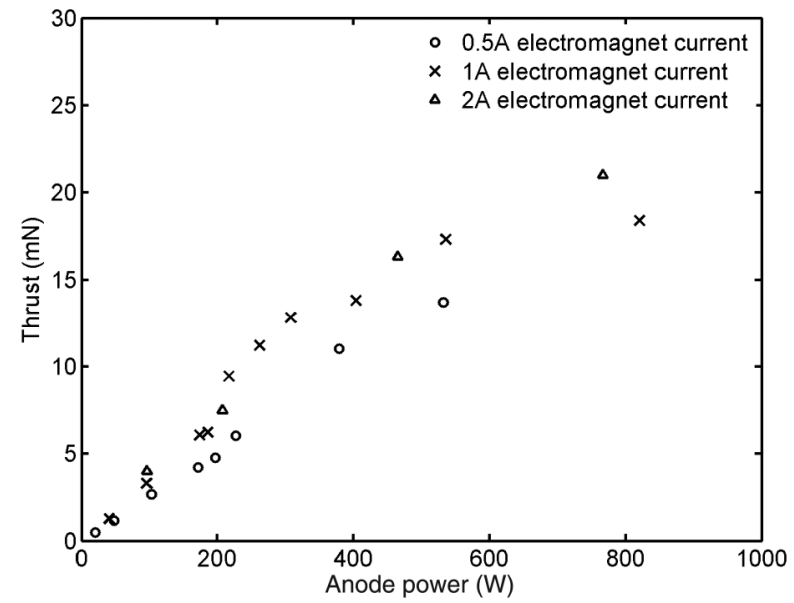

a)

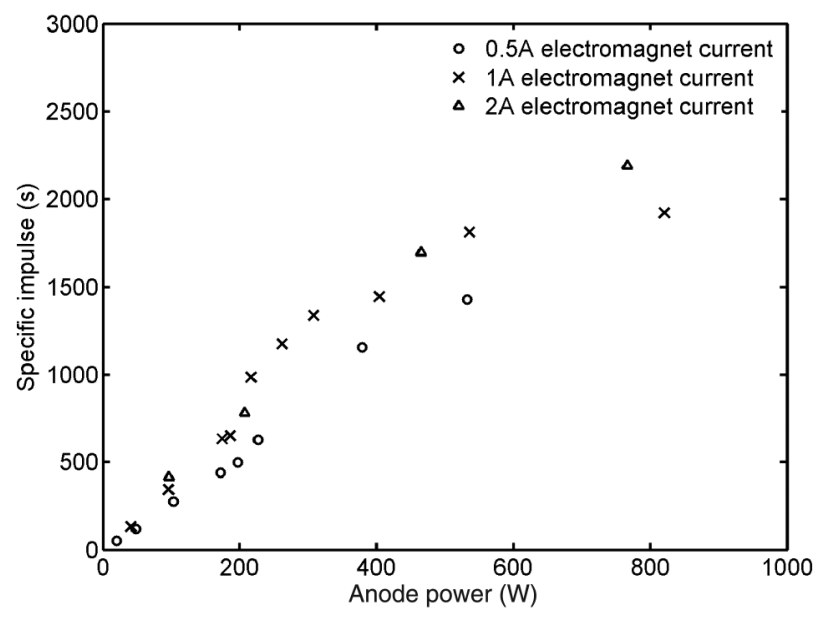

b)

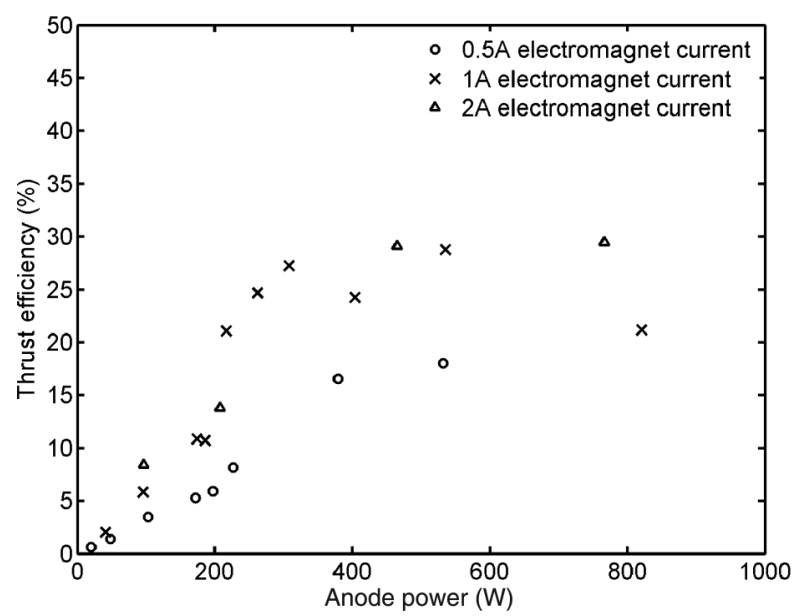

c)

Fig. 9 Performance values of the EM Halo thruster with anode power using xenon, at an anode flow rate of $10 \mathrm{sccm}$.

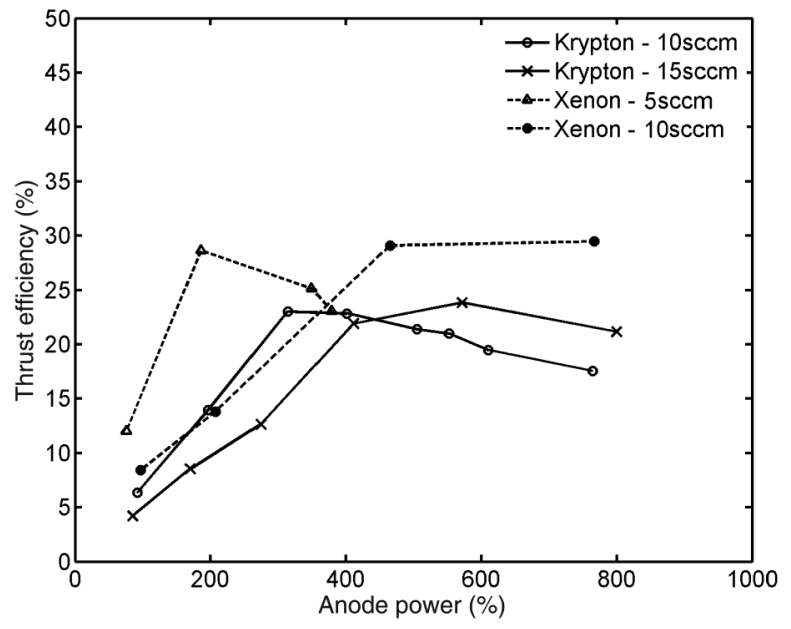

Fig. 10 Performance values of the EM Halo thruster vs anode power using xenon and krypton propellants; coil current is $2 \mathrm{~A}$ throughout.

increase with power up to a maximum of $12 \mathrm{mN}$ and $2000 \mathrm{~s}$, respectively, at approximately $800 \mathrm{~W}$. There is a small difference between the data obtained at different electromagnet currents, with lower current resulting in slightly lower thrust and specific impulse.

The anode efficiency demonstrates a peak in efficiency at approximately $400 \mathrm{~W}$. Increasing the electromagnet current results in a greater efficiency, with the greatest efficiency of $23 \%$ found at an anode power of $315 \mathrm{~W}$ and a coil current of $2 \mathrm{~A}$. All measures of performance decrease with decreasing coil current, particularly thrust efficiency.

Note that it was found experimentally that the thruster would heat up very quickly at above $600 \mathrm{~W}$, with sustained operation not possible. At the thrusters designed operating range of 200-400 W, the thruster demonstrating no noticeable overheating, operating well over 4-5 h.

Experiments were completed using xenon at anode flow rates of 5 and $10 \mathrm{sccm}$. Figure 9 illustrates the results at $10 \mathrm{sccm}$, again at electromagnet currents of $0.5,1$, and $2 \mathrm{~A}$. There appears to be a little difference between the performance of the thruster in the 1 and $2 \mathrm{~A}$ cases. The efficiency plateaus at $29 \%$ beyond around $300 \mathrm{~W}$, whereas thrust and $I_{\mathrm{sp}}$ continue to increase at higher powers at a relatively constant efficiency, up to a maximum of $21 \mathrm{mN}$ and $2190 \mathrm{~s}$ at $770 \mathrm{~W}$ in the $2 \mathrm{~A}$ case. All measures of performance decrease for the $0.5 \mathrm{~A}$ case as expected.

Figure 10 displays a comparison of thrust efficiencies achieved using xenon or krypton, at a fixed coil current of $2 \mathrm{~A}$. Performance is increased through the use of xenon, as expected through its lower ionization energy. For higher flow rates, the efficiency peaks at higher powers. This is especially true for using xenon, in which the efficiency peaks at $190 \mathrm{~W}$ at a flow rate of $5 \mathrm{sccm}$, compared to $470 \mathrm{~W}$ at a flow rate of $10 \mathrm{sccm}$.

\section{Conclusions}

The Halo thruster is a novel cusped-field thruster related to the CHT, with the addition of a toroidal cusp layer, or halo, close to the anode. The PM and electromagnet versions of the concept have been developed and successfully operated over a range of powers and propellant flow rates relevant to small satellite propulsion. Using a pendulum thrust balance, performance comparable to other cuspedfield concepts has been demonstrated for the EM variant, with measured thrust exceeding $20 \mathrm{mN}$, specific impulse exceeding $2000 \mathrm{~s}$, and efficiency approaching $30 \%$ using xenon, without extensive optimization. The majority of measurements exhibit a discharge voltage of less than $200 \mathrm{~V}$, lower than that of a typical CHT - this may have useful practical implications for power-processing hardware. The relatively low discharge voltage may constitute evidence of significant double ionization in the thruster, potentially brought about by the presence of the halo cusp layer. The presence of doubly (or triply) ionized propellant implies a higher change in ion velocity over 
a given potential drop, allowing an equivalent exit velocity to be achieved at lower applied voltages. Further experiments are required to establish the significance of the magnetic-cancellation region (the Halo) on the ionization and confinement of electrons within the acceleration channel, and any potential departures in the mechanism of operation from conventional CHT devices.

\section{Acknowledgments}

The authors would like to acknowledge the support of Airbus Defence and Space for the development of the Halo thruster, Surrey Satellite Technology Limited, and the general support of the Space Propulsion Lab at the Surrey Space Centre.

\section{References}

[1] Raitses, Y., and Fisch, N., "Parametric Investigations of a Nonconventional Hall Thruster," Physics of Plasmas, Vol. 8, No. 5, 2001, pp. 25792586. doi:10.1063/1.1355318

[2] Raitses, Y., Smirnov, A., and Fisch, N. J., "Cylindrical Hall Thrusters," AIAA Paper 2006-3245, 2006.

[3] Courtney, D. G., "Development and Characterization of a Diverging Cusped Field Thruster and a Lanthanum Hexaboride Hollow Cathode," M.S. Thesis, Massachusetts Inst. of Technology, Cambridge, MA, 2008.

[4] MacDonald, N. A., Cappelli, M. A., Gildea, S. R., Martinez-Sanchez, M., and Hargus, W. A., Jr., "Laser-Induced Fluorescence Velocity Measurements of a Diverging Cusped-Field Thruster," Journal of Physics D: Applied Physics, Vol. 44, No. 29, 2011, Paper 295203. doi:10.1088/0022-3727/44/29/295203

[5] Kornfeld, G., Koch, N., and Harmann, H.-P., "Physics and Evolution of HEMP-Thrusters," Proceedings of the 30th International Electric Propulsion Conference, 2007, pp. 17-20.

[6] Kornfeld, G., Koch, N., and Harmann, H., "New Performance and Reliability Results of the Thales HEMP Thruster," Proceedings of the 4th International Spacecraft Propulsion Conference, 2004, p. 39.

[7] Kornfeld, G., Coustou, G., and Emsellem, G., Google Patents, 2006.

[8] Koch, N., Schirra, M., Weis, S., Lazurenko, A., van Reijen, B., Haderspeck, J., Genovese, A., Holtmann, P., Schneider, R., and Matyash, K., "The HEMPT Concept-A Survey on Theoretical
Considerations and Experimental Evidences," Proceedings of the 32nd International Electric Propulsion Conference, Sept. 2011, p. 15.

[9] Wantock, T., Ryan, C., Harle, T., and Knoll, A., "Initial Performance Characterisation of a Plasma Thruster Employing Magnetic Null Regions," Proceedings of the 34th International Electric Propulsion Conference, Electric Rocket Propulsion Soc., Kobe, Japan, 2015.

[10] Longmier, B., Baalrud, S., and Hershkowitz, N., "Nonambipolar Electron Source," Review of Scientific Instruments, Vol. 77, No. 11, 2006, Paper 113504. doi:10.1063/1.2393164

[11] Gildea, S. R., Martinez-Sanchez, M., Nakles, M. R., and Hargus, W. A., Jr., "Experimentally Characterizing the Plume of a Divergent CuspedField Thruster," U.S. Air Force Research Lab. AFRL-RZ-ED-TP-2009324, Edwards Air Force Base, CA, Aug. 2009.

[12] Courtney, D. G., and Martinez-Sanchez, M., "Diverging Cusped-Field Hall Thruster (DCHT)," Proceedings of the 30th International Electric Propulsion Conference, 2007.

[13] Matlock, T., Hu, F., and Martinez-Sanchez, M., "Controlling Plume Divergence in a Cusped-Field Thruster," Proceedings of the 32nd International Electric Propulsion Conference, 2011.

[14] Smirnov, A., Raitses, Y., and Fisch, N. J., "Experimental and Theoretical Studies of Cylindrical Hall Thrusters," Physics of Plasmas, Vol. 14, 2007, Paper 057106.

[15] Polzin, K. A., Raitses, Y., Gayoso, J. C., and Fisch, N. J., "Comparisons in Performance of Electromagnet and Permanent-Magnet Cylindrical Hall-Effect Thrusters," Proceedings of the 46th AIAA/ASME/SAE/ASEE Joint Propulsion Conference and Exhibit, 2010.

[16] Knoll, A., Harle, T., Lappas, V., and Pollard, M., "Experimental Performance Characterization of a Two-Hundred-Watt Quad Confinement Thruster," Journal of Propulsion and Power, Vol. 30, No. 6, 2014, pp. 1445-1449. doi:10.2514/1.B35218

[17] Pottinger, S., Lamprou, D., Knoll, A., and Lappas, V., "Impact of Plasma Noise on a Direct Thrust Measurement System," Review of Scientific Instruments, Vol. 83, No. 3, 2012, Paper 033504. doi: $10.1063 / 1.3692740$

[18] Pollard, M., and Lamprou, L., "The Design, Development, Manufacture and Test of an Electric Propulsion System for Small Spacecraft," Proceedings of the Space Propulsion Conference, 2012.

Prof. A. D. Gallimore Associate Editor 


\section{Queries}

1. AU: Please check that the copyright (C) type is correct. Please note that the code will be added upon publication.

2. AU: Per journal guidelines, acronyms and abbreviations can only be used in the Abstract if they meet all of the following criteria: 1) they are more than 12 characters long; 2) they are used more than once in the Abstract; and 3) they are redefined upon first usage in the main text.

3. AU: Minor syntax adjustments were made throughout; please read closely to confirm that your meaning was retained.

4. AU: The sentence beginning, "Secondly, the cusp structure..." seems unclear and incomplete. Please review and edit as necessary.

5. AU: Per journal guidelines, the use of the word "in which" is preferred over "where," unless it denotes a location. Please confirm all changes made are correct, or correct if necessary.

6. AU: AIAA has not indicated color use for your paper. Please confirm that your paper should be grayscale and that all figures are satisfactory. If any replacement figures are needed, please send them in .eps or.tiff format to aiaa-proofs@ beacon.com and use code JPP-B36091 in the subject line. Formats of .jpg, .doc, or .pdf can be used with some loss of quality.

7. AU: Please note that figures must be cited in numerical order within the article's text. Figure 3 was cited before Fig. 2. Please review and edit as necessary.

8. AU: Please note that acronyms must be used more than once to remain in the paper.

9. AU: AIAA prefers that figure captions be 3-12 words long. The captions to Figs. 2 and $4-10$ far exceed this limit. Please reduce the length of these captions to no more than 12 words and/or incorporate the information into the text.

10. AU: In Fig. 2, "Magentic" has been changed to "Magnetic." Please confirm your meaning was retained.

11. AU: In the sentence beginning, "The error on the thrust ...," "relative consistent" has been changed to "relatively consistent." Please confirm your meaning was retained, or correct if necessary.

12. AU: The sentence beginning, "The sensitivity of the thruster..." seems unclear and incomplete. Please review and edit as necessary.

13. AU: In the sentence beginning, "At the thrusters designed operating range ...," please check if "thrusters designed" should be "thruster design."

14. AU: The use of the word "although" or "whereas" is preferred over "while/whilst," unless it means "at the same time," per journal guidelines. Please confirm all changes made are correct, or correct if necessary.

15. AU: Please provide the month of publication of Ref. [2].

16. AU: Please provide the publisher name and location of Refs. [5, 6, 8].

17. AU: Please confirm that Refs. $[6,8]$ are one-page articles. If there is more than one page, provide the complete page range.

18. AU: Please provide the patent number and date of Ref. [7].

19. AU: Please provide the page range of Ref. [9]. 
20. AU: Missing important details of Ref. [11] have been supplied, per online check. Please check and correct if necessary.

21. AU: References [11-15] are not cited in the main text. Please cite the references in the main text in numerical order or remove the references.

22. AU: If Refs. $[12,13,15,18]$ are published proceedings, please provide the name and location of the publisher (NOT of the conference host), and the page range. If they are conference papers, please provide the paper number and the organizer's name. If they are CD-ROMs, please provide the name and location of the CD-ROM producer.

23. AU: Please provide the issue number or month of publication of Ref. [14]. 